\title{
Stereotactic body radiotherapy (SBRT) for pulmonary metastases from renal cell carcinoma-a multicenter analysis of the German working group "Stereotactic Radiotherapy"
}

\author{
Juliane Hoerner-Rieber ${ }^{1,2}$, Marciana Duma ${ }^{3}$, Oliver Blanck ${ }^{4}$, Guido Hildebrandt ${ }^{5}$, Andrea Wittig ${ }^{6}$, Fabian \\ Lohaus $^{7,8,9}$, Michael Flentje ${ }^{10}$, Frederick Mantel ${ }^{10}$, Robert Krempien ${ }^{11}$, Michael J. Eble ${ }^{12}$, Klaus Henning
} Kahl $^{13}$, Judit Boda-Heggemann ${ }^{14}$, Stefan Rieken ${ }^{1,2}$, Matthias Guckenberger ${ }^{15}$

${ }^{1}$ Department of Radiation Oncology, University Hospital Heidelberg, Heidelberg, Germany; ${ }^{2}$ National Center for Radiation Oncology (NCRO), Heidelberg Institute for Radiation Oncology (HIRO), Heidelberg, Germany; ${ }^{3}$ Department of Radiation Oncology, Technical University Munich, Germany; ${ }^{4}$ Department of Radiation Oncology, UKSH Universitätsklinikum Schleswig-Holstein, Kiel, Germany; ${ }^{5}$ Department of Radiation Oncology, University of Rostock, Rostock, Germany; 'Department of Radiotherapy and Radiation Oncology, Philipps-University Marburg, University Hospital Giessen and Marburg, Germany; ${ }^{7}$ Department of Radiation Oncology, Medical Faculty and University Hospital C.G. Carus, Technical University Dresden, Germany; ${ }^{8}$ German Cancer Research Center (DKFZ), Heidelberg and German Cancer Consortium (DKTK) partner site Dresden, Germany; ${ }^{9}$ OncoRay - National Center for Radiation Research in Oncology, Faculty of Medicine and University Hospital Carl Gustav Carus, Technische Universität Dresden, Helmholtz-Zentrum Dresden-Rossendorf, Germany; ${ }^{10}$ Department of Radiation Oncology, University Hospital Wuerzburg, Germany; ${ }^{11}$ Department of Radiation Oncology, Helios Klinikum Berlin Buch, Berlin, Germany; ${ }^{12}$ Department of Radiation Oncology, University Hospital Aachen, Aachen, Germany; ${ }^{13}$ Department of Radiation Oncology, Hospital Augsburg, Germany; ${ }^{14}$ Department of Radiation Oncology, University Medical Center Mannheim, University of Heidelberg, Germany; ${ }^{15}$ Department of Radiation Oncology, University Hospital Zurich, University of Zurich, Switzerland

Contributions: (I) Conception and design: J Hoerner-Rieber, M Guckenberger; (II) Administrative support: J Hoerner-Rieber, M Guckenberger; (III) Provision of study materials or patients: All authors; (IV) Collection and assembly of data: All authors; (V) Data analysis and interpretation: J Hoerner-Rieber, M Guckenberger; (VI) Manuscript writing: All authors; (VII) Final approval of manuscript: All authors.

Correspondence to: Dr. Juliane Hoerner-Rieber. Department of Radiation Oncology, University Hospital Heidelberg, INF 400, 69120 Heidelberg, Germany. Email: juliane.rieber@med.uni-heidelberg.de.

Background: Renal cell carcinoma (RCC) is traditionally considered to be radioresistant. Radiotherapy response rates are believed to improve with hypofractionated, high dose stereotactic body radiotherapy (SBRT). However, limited data exist regarding the role of SBRT in the treatment of pulmonary metastases.

Methods: The working group "Stereotactic Radiotherapy" of the German Society of Radiation Oncology analyzed its multi-institutional database of more than 700 patients who received SBRT for pulmonary metastases. Treatment was performed at 10 centers between 2001 and 2016. Patients with metastatic RCC were included in the study. Tumor characteristics, treatment details, and follow-up data including survival, local control (LC), distant metastases, and toxicity were evaluated.

Results: A total of 46 RCC patients treated with SBRT for 67 lung metastases were identified, who received a median total biologically effective dose $\left(\mathrm{BED}_{\text {iso }}\right)$ at planning target volume (PTV) isocenter of 117.0 Gy (range, 48.0-189.0 Gy). A median fractional dose of 20.8 Gy at isocenter (range, 6.0-37.9 Gy) was administered in a median number of 3 fractions (1-8 fractions). After a median follow-up time of 28.3 months for all patients, 1 - and 3-year LC rates were $98.1 \%$ and $91.9 \%$, with corresponding 1 - and 3-year overall survival (OS) of $84.3 \%$ and $43.8 \%$, respectively. Pulmonary metastases treated with BEDiso $\geq 130$ Gy showed a trend for superior LC $(\mathrm{P}=0.054)$. OS was significantly improved in both uni- and multivariate analysis for patients with higher Karnofsky performance scale, lower maximum pulmonary metastasis diameter and lack of post-SBRT systemic therapy due to progression ( $\mathrm{P}=0.014 ; \mathrm{P}=0.049 ; \mathrm{P}=0.006)$. Only mild acute and late toxicity was reported. 
Conclusions: SBRT for pulmonary metastases from RCC was associated with low treatment-associated toxicity, promising survival, and excellent LC, especially in those patients receiving a $\mathrm{BED}_{\text {iso }} \geq 130$ Gy.

Keywords: Renal cell carcinoma (RCC); lung metastases; stereotactic body radiotherapy (SBRT); radioresistance; extracranial stereotactic radiotherapy

Submitted Aug 09, 2017. Accepted for publication Oct 10, 2017. doi: $10.21037 /$ jtd.2017.10.108

View this article at: http://dx.doi.org/10.21037/jtd.2017.10.108

\section{Introduction}

Renal cell carcinoma (RCC) represents $2-3 \%$ of adult cancers in the European Union and accounts for approximately 34,700 deaths per year $(1,2)$. Although 5 -year survival for all patients is approximately $74 \%$, survival rates are highly dependent on disease stage, declining to $67 \%$ when locoregional disease (stage III) is detected and $12 \%$ in patients with distant metastases $(3,4)$. Most patients are diagnosed at an early stage, yet nearly one third of these patients will suffer from local or distant relapse in the course of their disease, and a similar proportion of patients present with metastatic spread at first diagnosis $(5,6)$. Lung metastases are the most common presentation of distant disease, comprising $11-75 \%$ of cases, while hepatic metastases occur in $20-40 \%$ of patients (7-12).

When distant metastases are diagnosed, systemic treatment with targeted agents is usually administered (3). However, an oligo-metastatic state has been hypothesized, where systemic therapy and potential toxicity might be postponed by applying local treatment strategies to all evident tumor lesions (13). Furthermore, an objective response has been reported in $20-40 \%$ of patients, but complete response was only detected in $1-3 \%$ of cases with current targeted drugs (13-16). Consequently, in cases of oligoprogression, local ablative treatment to all synchronous and metachronous metastases, when technically feasible and clinically appropriate, might represent a potentially curative approach (13).

Surgical series for pulmonary metastasectomy as a local treatment method of RCC patients report excellent local control (LC) in case of complete resection and promising 3 - and 5-year survival rates of $49-66 \%$ and $31-58 \%$, respectively (17-27). However, some RCC patients are medically inoperable due to reduced performance status or comorbidities and some pulmonary metastases are technically not accessible or resectable. For these patients, stereotactic body radiotherapy (SBRT) which utilizes highly conformal ablative local doses to the tumor while sparing surrounding organs, has shown encouraging results (5,28-30). However, available reports concerning SBRT for RCC metastases have pooled heterogeneous data from various tumor locations (lung, bone, lymph node, brain, liver, etc.) with different sensitivities to high-dose radiation (5,6,28-33). This multi-institutional study reports the feasibility, safety, and efficacy of SBRT for pulmonary RCC metastases.

\section{Methods}

The working group "Stereotactic Radiotherapy" of the German Society of Radiation Oncology (DEGRO) analyzed its retrospective multi-institutional database including more than 700 patients treated with SBRT for more than 900 pulmonary metastases. Detailed description of the database has been previously published (34-36). For the current study, an update of the database was performed in April 2017. Patient data from institutions that did not participate in this database update were excluded from this study. In total, 46 patients with histologically confirmed RCC treated with SBRT for 67 pulmonary metastases at 10 different German centers between 2001 and 2016 were analyzed. Each center compiled patient characteristics, treatment details, and outcome data in an anonymized electronic file and delivered it to the coordinating center, where a pooled database was created. The analysis was approved by the Ethics committee of the University Hospital Heidelberg (S-280/2014).

Pulmonary SBRT was utilized if patients were classified medically inoperable, diagnosed with unresectable lung metastases or refused surgical resection. Fluoro-deoxyglucose positron emission tomography (FDG-PET) imaging was only performed in five patients and biopsy confirmation was performed when the origin of the pulmonary lesion was in question $(n=7)$. All centers used risk-adapted fractionation schemes adjusting the number 
of fractions and single-fraction doses to tumor size and location (peripheral vs. central). Metastases were classified to be "peripheral" or "central" according to the Radiation Therapy Oncology Group (RTOG) definition $(37,38)$. Treatment-related toxicity was categorized according to CTCAE v4.0.

For correlating radiation doses with clinical results, the biological effective dose (BED) was determined assuming: an $\alpha / \beta$ ratio of $10 \mathrm{~Gy}$ for the pulmonary metastases. BED was calculated using the linear-quadratic model (39):

$\operatorname{BED}(\mathrm{Gy})=$ fractional dose $\times$ number of fractions $\left(1+\frac{\text { fractional dose }}{\alpha / \beta}\right)$

LC, overall survival (OS) and distant control (DC) were estimated using the Kaplan-Meier method. While LC was calculated for each individual pulmonary metastasis, OS and DC were determined following SBRT for the first, index pulmonary lesion if several pulmonary metastases were treated. LC was defined as no progressive disease within the high-dose area. Recurrences distant to the treated pulmonary metastasis in the same lobe were not classified as local but as distant failure.

Survival curves were compared between groups in univariate analysis applying the log-rank test or cox regression analysis. Receiver operating characteristics (ROC) curves and the Youden's index were applied to determine the optimal cut-off for BED at planning target volume (PTV) isocenter $\left(\mathrm{BED}_{\mathrm{ISO}}\right)$. Multivariate cox models were performed including all variables which were statistically significant in univariate analysis. Multivariate analysis was not performed for LC, as no significant prognostic factor was identified in univariate analysis. LC, OS and DC were analyzed from the start of SBRT until the event of interest or the last follow-up visit. A $\mathrm{P} \leq 0.05$ was considered statistically significant. All statistical analyses were performed with SPSS software (version 20.0).

\section{Results}

\section{Patient and treatment characteristics}

This multi-institutional analysis included data from 10 centers with extensive lung SBRT experience, of which seven were university hospitals. In total, data were collected from 46 patients treated with SBRT for 67 pulmonary metastases. Most patients were treated for one lung metastasis (median: 1 metastasis, range, 1-7 metastases). All patients were diagnosed with biopsy-proven metastatic
RCC and had in median one additional metastasis (range, 0-15) besides the treated pulmonary lesion(s). Additional metastases were located in $77.1 \%$ in the lung. Of these $48.6 \%$ were treated with resection and $31.4 \%$ underwent additional SBRT. Overall, $83 \%$ of patients received definitive treatment with curative intent (SBRT, definitive radiotherapy or surgery, sometimes in combination with systemic therapy) to all known sites of metastasis, while the remaining $17 \%$ were treated with systemic therapy including chemotherapy, immunotherapy, or targeted agents. In total, 22 patients received systemic therapy prior to SBRT. Of these, chemotherapy was administered in two patients, followed by immunotherapy in five patients and tyrosine kinase inhibitors in 15 patients. SBRT to pulmonary metastases was applied at a median 73.8 months (range, 3.2 months-28.8 years) following diagnosis of the primary tumor. The time interval between diagnosis of the pulmonary metastasis and SBRT treatment was found to be 2.7 months (0.2-83.1 months) in median. Detailed patient and treatment characteristics are illustrated in Tables 1 and 2.

\section{OS, LC and systemic progression}

Median follow-up time for all patients was 28.3 months (range, 0.8-133.8 months) with 1- and 3-year LC rates of $98.1 \%$ and $91.9 \%$, respectively (Figure $1 A$ ). Only 3 local relapses $(4.5 \%)$ were detected during the follow-up period with a median time to local failure of 26.2 months (range, 10.5-27.7 months). Univariate analysis revealed a trend for increased LC when the $\mathrm{BED}_{\text {ISO }} \geq 130$ Gy $(\mathrm{P}=0.054)$ (Figure 1B) (Table 3). Pulmonary metastases treated with a $\mathrm{BED}_{\text {ISO }}<130$ Gy resulted in a 3-year LC of $83.9 \%$, while receipt of a $\mathrm{BED}_{\mathrm{ISO}} \geq 130$ Gy was associated with a 3 -year LC of $100 \%$.

One- and 3-year distant metastases free survival was found to be $45.7 \%$, and $17.0 \%$. Distant disease progression was the most common pattern of failure, with $69.6 \%$ of patients $(n=32)$ developing new, out-of-field metastases. Distant metastases were mostly detected in the lung $(\mathrm{n}=26)$, the bone $(n=4)$, the liver $(n=4)$, the brain $(n=4)$, and other locations $(n=4)$.

One- and 3-year OS was $84.3 \%$ and $43.8 \%$ respectively (Figure 2). Karnofsky performance score, maximum pulmonary metastasis diameter and the lack of admission of systemic therapy after SBRT due to disease progression were identified as prognostic factors for OS in univariate analysis $(\mathrm{P}=0.010 ; \mathrm{P}=0.011, \mathrm{P}=0.001)$ (Table 3). Furthermore, the presence of metachronous metastases was associated with a trend towards improved OS compared to synchronous 
Table 1 Baseline patient and lesion characteristics for 46 patients treated with SBRT for 67 pulmonary metastases

\begin{tabular}{|c|c|c|c|c|c|}
\hline Factors & No. of patients/lesions & $\%$ & Median & Minimum & Maximum \\
\hline Age (yr) & 46 & & 68.5 & 35.4 & 85.1 \\
\hline Sex & 46 & & & & \\
\hline Male & 34 & 73.9 & & & \\
\hline Pretreatment performance scale (Karnofsky index) (\%) & 46 & & 90 & 60 & 100 \\
\hline Cancer stage at first diagnosis & 46 & & & & \\
\hline Stage I & 10 & 21.8 & & & \\
\hline Stage II & 7 & 15.2 & & & \\
\hline Histology & 46 & & & & \\
\hline Clear cell & 33 & 71.7 & & & \\
\hline Other & 8 & 17.4 & & & \\
\hline Unavailable & 5 & 10.9 & & & \\
\hline Grade & 46 & & & & \\
\hline Grade 1 & 7 & 15.2 & & & \\
\hline Grade 2 & 24 & 52.2 & & & \\
\hline Grade 3 & 5 & 10.9 & & & \\
\hline Peripheral & 54 & 80.6 & & & \\
\hline Number of metastases & 67 & & & & \\
\hline Single & 12 & 17.9 & & & \\
\hline Multiple & 55 & 82.1 & & & \\
\hline Time to metastasis & 67 & & & & \\
\hline Synchronous & 3 & & & & \\
\hline Metachronous & 64 & & & & \\
\hline Time interval between SBRT and first tumor diagnosis (years) & 67 & & 6.15 & 0.27 & 28.8 \\
\hline Systemic therapy before SBRT & 67 & & & & \\
\hline Yes & 35 & 52.2 & & & \\
\hline No & 32 & 47.8 & & & \\
\hline
\end{tabular}

Table 1 (continured) 
Table 1 (continured)

\begin{tabular}{|c|c|c|c|c|c|}
\hline Factors & No. of patients/lesions & $\%$ & Median & Minimum & Maximum \\
\hline Yes & 13 & 19.4 & & & \\
\hline No & 54 & 89.6 & & & \\
\hline Systemic therapy after SBRT & 67 & & & & \\
\hline No & 40 & 59.7 & & & \\
\hline
\end{tabular}

SBRT, stereotactic body radiotherapy.

Table 2 SBRT treatment patterns for 67 lung metastases

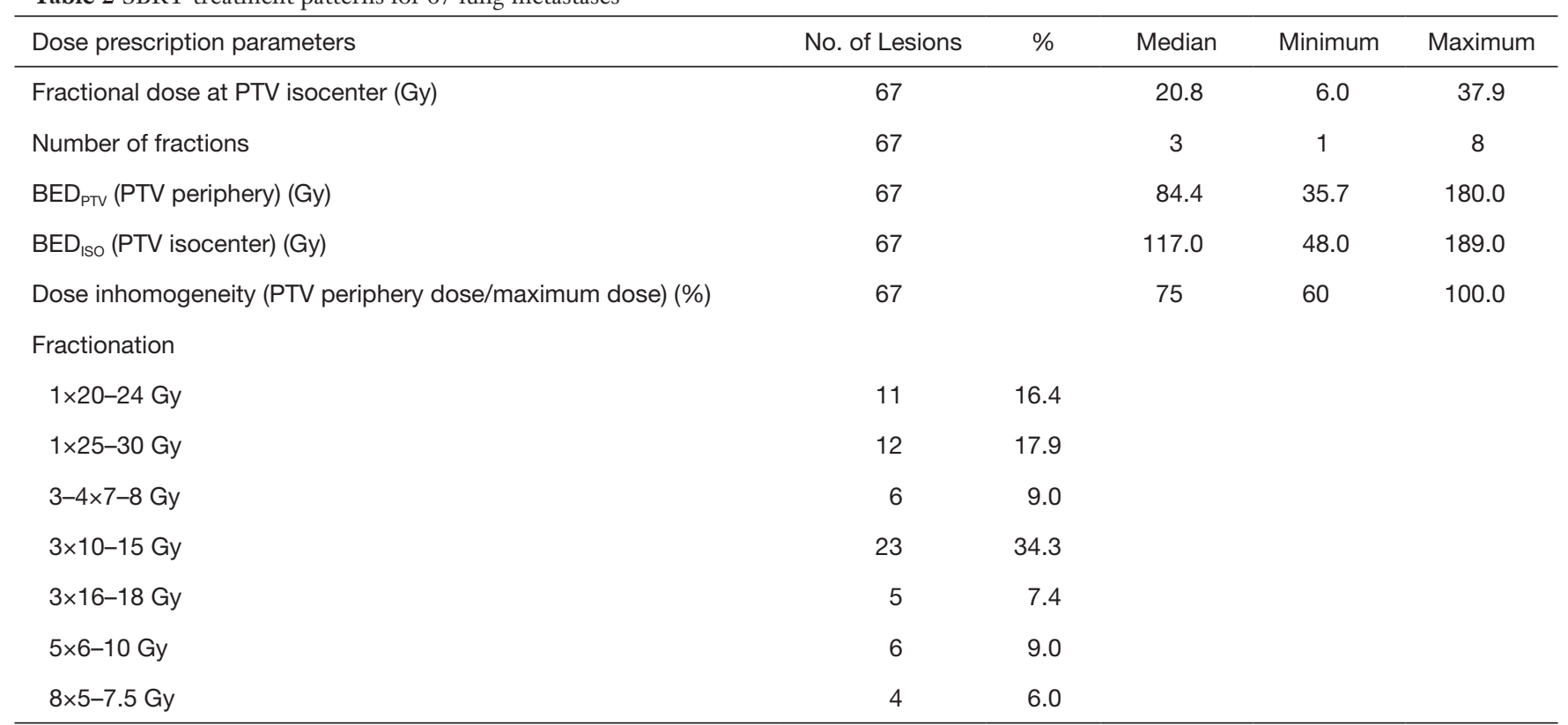

SBRT, stereotactic body radiotherapy; PTV, planning target volume; BED, biological effective dose.

lesions $(\mathrm{P}=0.055)$. Patients receiving definitive treatment to all metastatic lesions had non-significantly improved survival compared to patients who were treated with systemic therapy and SBRT-debulking of disease with 3-year OS-rates of $50.5 \%$ and $14.6 \%$, respectively $(\mathrm{P}=0.077)$. Multivariate analysis revealed higher Karnofsky performance score, lower maximum pulmonary metastasis diameter as well as no admission of systemic therapy after SBRT as independent prognostic factors for $\mathrm{OS}(\mathrm{P}=0.014 ; \mathrm{P}=0.049 ; \mathrm{P}=0.006)$.

\section{Toxicity}

Acute toxicity was mild with only four patients developing radiation induced common terminology criteria for adverse events (CTCAE) grade II pneumonitis. Three of these four patients were treated with a second course of SBRT to another pulmonary metastasis within 14 days of initial SBRT. No grade III-V pneumonitis was reported. One patient died 0.9 months following SBRT; consequently 30- and 60-day death rates were $1.5 \%$. The death of this patient was not attributable to SBRT treatment, as it was secondary to perforated diverticulitis with peritonitis. Three patients developed further grade II+ toxicity: pulmonary fibrosis (1 patient), atelectasis (1 patient) and pleural effusion (1 patient). Higher toxicity rates were not observed in patients who received systemic therapy before SBRT. 

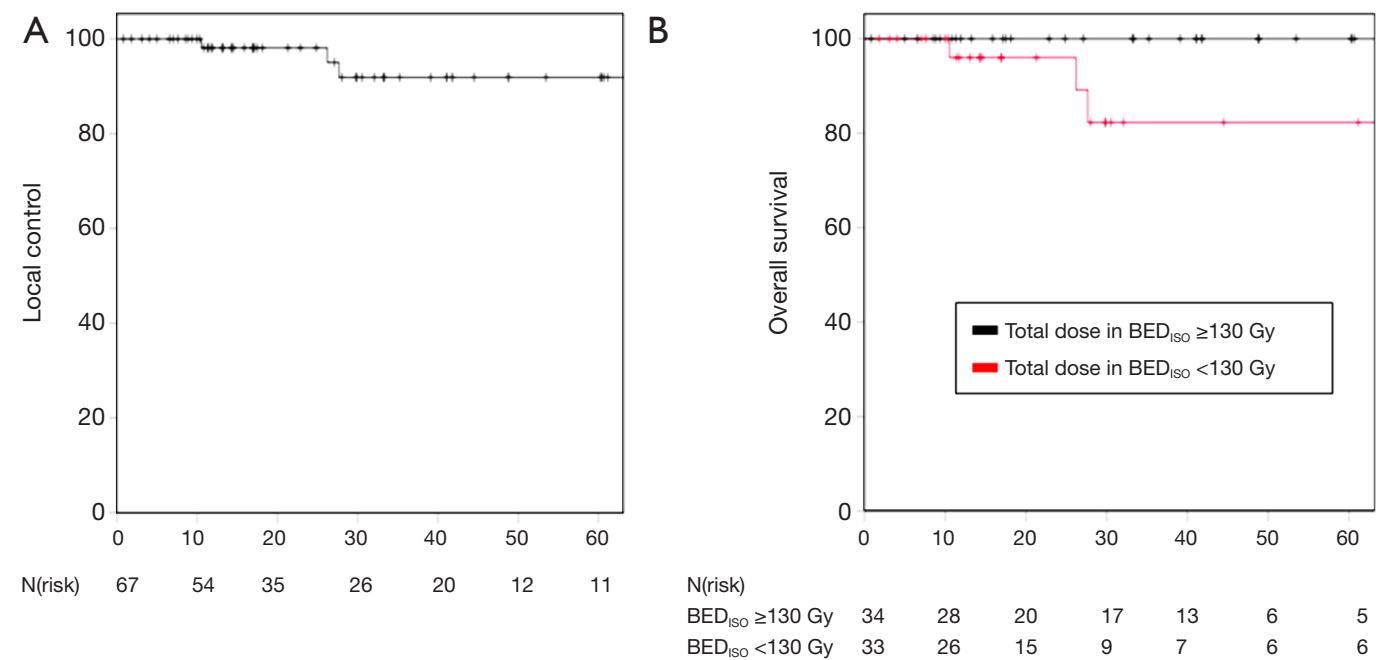

Figure 1 Outcome analysis. (A) Local control (LC) following SBRT for 67 lung metastases from RCC; (B) metastases treated with BED ISO $\geq 130$ Gy showed a tendency for superior LC $(\mathrm{P}=0.054)$. SBRT, stereotactic body radiotherapy; RCC, renal cell carcinoma.

Table 3 Univariate analysis of prognostic factors influencing LC and OS

\begin{tabular}{|c|c|c|c|c|c|c|}
\hline \multirow{2}{*}{ Factors } & \multicolumn{3}{|c|}{ LC } & \multicolumn{3}{|c|}{ OS } \\
\hline & $\mathrm{HR}$ & $95 \% \mathrm{Cl}$ & $\mathrm{P}$ & $\mathrm{HR}$ & $95 \% \mathrm{Cl}$ & $\mathrm{P}$ \\
\hline Sex (female vs. Male) & 0.281 & $(0.025,3.107)$ & 0.130 & 0.622 & $(0.233,1.659)$ & 0.343 \\
\hline Age & 1.094 & $(0.920,1.303)$ & 0.310 & 1.002 & $(0.953,1.053)$ & 0.946 \\
\hline Karnofsky performance scale & 0.977 & $(0.879,1.087)$ & 0.673 & 0.962 & $(0.933,0.991)$ & 0.010 \\
\hline Time to metastasis (synchronous vs. Metachronous) & 0.542 & $(0.049,5.998)$ & 0.618 & 0.425 & $(0.177,0.999)$ & 0.055 \\
\hline Number of metastases (solitary vs. multiple) & 3.343 & $(0.303,36.876)$ & 0.325 & 1.044 & $(0.431,2.525)$ & 0.924 \\
\hline Maximum pulmonary metastasis diameter & 1.276 & $(0.588,2.767)$ & 0.537 & 1.339 & $(1.068,1.677)$ & 0.011 \\
\hline Total dose in $\mathrm{BED}_{\mathrm{ISO}}:<130 \mathrm{~Gy} ; \geq 130 \mathrm{~Gy}$ & 0.012 & $(0.001,37.987)$ & 0.054 & 1.259 & $(0.583,2.746)$ & 0.553 \\
\hline Definitive treatment to all further metastases (yes vs. No) & $\mathrm{n}$ & $\mathrm{n}$ & $\mathrm{n}$ & 2.334 & $(0.911,5.977)$ & 0.077 \\
\hline Systemic therapy in general (no vs. yes) & 0.666 & $(0.060,7.352)$ & 0.740 & 0.557 & $(0.252,1.233)$ & 0.149 \\
\hline Systemic therapy before SBRT (no vs. yes) & 2.133 & $(0.193,23.574)$ & 0.537 & 0.673 & $(0.314,1.444)$ & 0.309 \\
\hline Systemic therapy 4 weeks before SBRT (no vs. yes) & 3.641 & $(0.161,36.767)$ & 0.727 & 0.392 & $(0.139,1.100)$ & 0.075 \\
\hline Systemic therapy after SBRT (no vs. yes) & 0.155 & $(0.013,1.771)$ & 0.133 & 0.190 & $(0.069,0.527)$ & 0.001 \\
\hline Systemic therapy 4 weeks after SBRT (no vs. yes) & 0.721 & $(0.293,1.322)$ & 0.290 & 0.803 & $(0.622,1.038)$ & 0.094 \\
\hline
\end{tabular}

The variables sex, histology, grade, time of metastasis, number of metastases; tumor location and total dose in $B E D_{\text {Iso }}</ \geq 130$ Gy were analyzed as categorical variables, while the other variables were taken as continuous variables for analysis. $n$, calculation of confidence intervals was not possible due to too few cases and events in each single group. OS, overall survival; LC, local control; HR, hazard ratio; BED, biological effective dose; PTV, planning target volume; SBRT, stereotactic body radiotherapy. 


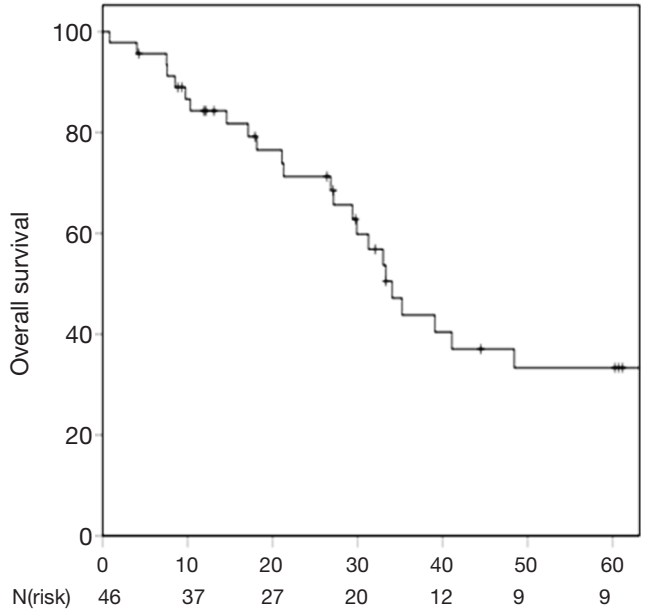

Figure 2 Overall survival (OS) after SBRT of 67 pulmonary metastases in $46 \mathrm{RCC}$ patients. SBRT, stereotactic body radiotherapy; RCC, renal cell carcinoma.

\section{Discussion}

RCC is traditionally perceived to be a radioresistant malignancy with a limited role for radiotherapy in the management of localized disease (1). Although prospective trials investigating the role of radiotherapy in the neoadjuvant and adjuvant settings demonstrated improved LC, these findings have not translated into increased survival $(40,41)$. Contrarily, smaller retrospective studies have suggested a potential advantage for adjuvant radiotherapy with larger tumors (pT3) or positive resection margins $(40,42,43)$. Nevertheless, currently radiotherapy is primarily used for palliation of symptoms in metastatic RCC (3).

The efficacy of radiotherapy is highly dependent on applied doses and fractionation schemes. While RCC tumors appear to be radioresistant using conventionally fractionated radiotherapy, preclinical data suggest increased radiosensitivity of human RCC xenografts in nude mice when applying ablative, hypofractionated radiotherapy (44). In the clinical setting, dose escalation has also been shown to overcome radioresistance in RCC tumors: Zelefsky and colleagues reported 3-year LC of 88\% following SBRT with high fractional doses for extracranial RCC metastases, while LC dropped to only about $20 \%$ when lower doses were used (30). A few other reports have also revealed LC rates of about $80-90 \%$ for extracranial SBRT in RCC patients $(5,6,28,29,31,33)$. However, due to the limited number of RCC patients treated with SBRT, these reports combined data form different metastatic sites of including lung, bone, liver, lymph nodes or even primary RCC tumors $(5,6,28,29,31,33)$.

As LC and treatment-related toxicity following SBRT are known to be highly dependent on treatment location, it is essential to analyze different SBRT treatment locations separately. To our knowledge, this analysis is the first study focusing on SBRT for solely pulmonary metastases from RCC. We observed excellent 1- and 3-year LC rates of 98.1\% and $91.9 \%$ following SBRT of these metastases with low rates of treatment-associated toxicity.

In general, the most important factor for achieving optimal LC following SBRT is a sufficiently high BED. For lung and liver SBRT, doses above 100 Gy BED PTV $_{\text {(PTV }}$ minimum dose) are recommended, while lower doses seem sufficient for spinal metastases $(35,36,45-47)$. A recently published report by Wang et al. investigated SBRT for a wide variety of extracranial RCC metastases, including bone (38.8\%), abdominal (28.0\%) and thoracic (20\%) lesions, demonstrating significantly improved LC if a BED $\mathrm{PTV}_{\text {of }}$ 98.7 Gy or higher was applied (28). Altoos et al. compared SBRT $(\mathrm{n}=36)$ and conventionally fractionated radiotherapy $(n=17)$ for the treatment of thoracic, abdominal or soft tissue RCC metastases and also reported a $\mathrm{BED}_{\mathrm{PTV}}$ $\geq 100$ Gy to be associated with significantly superior LC. In our study, excellent LC rates exceeding $90 \%$ after 3 years for RCC pulmonary metastases were detected, despite a median BED $_{\text {PTV }}$ of 84.4 Gy. As in other studies, we also observed a dose-response relationship: if pulmonary metastases received a $\mathrm{BED}_{\text {Iso }} \geq 130$ Gy at $\mathrm{PTV}$ isocenter, there was a strong trend for improved LC $(\mathrm{P}=0.054)$. Notably, only three local recurrences were identified in our cohort and hence this result has to be interpreted with caution. Due to the very low number of local relapses detected in this study, consequently analysis of further potential prognostic factors regarding LC was highly limited (Table 3) and results have to be regarded as preliminary. Clearly, larger, multi-center studies are needed to further clarify the impact of suspected prognostic factors.

To date, only smaller and retrospective surgical series for pulmonary metastasectomy in RCC patients have reported good 3- and 5-year survival rates of 49-66\% and $31-58 \%$, respectively (Table 4) (17-27). In this study, estimated 3 - and 5 -year OS rates with $44 \%$ and $33 \%$ were slightly lower compared to these surgical series. However, comparison between these studies is limited, as nearly all patients in this study were classified as medically inoperable due to comorbidities or higher age. While median age was 68.5 years in this study, reported median 
Table 4 Surgical series for pulmonary metastasectomy in RCC patients

\begin{tabular}{|c|c|c|c|c|c|}
\hline Study & Year & Treatment years & Number of patients (n) & 3-year OS (\%) & 5 -year OS (\%) \\
\hline Pfannschmidt et al. (19) & 2002 & 1985-1999 & 191 & - & 37 \\
\hline Murthy et al. (18) & 2005 & 1986-2001 & 92 & 49 & 31 \\
\hline Hofmann et al. (17) & 2005 & 1975-2003 & 64 & - & 33 \\
\hline Kanzaki et al. (22) & 2010 & 1973-2008 & 48 & 60 & 47 \\
\hline Meimarakis et al. (23) & 2011 & 1986-2006 & 202 & - & 39 \\
\hline Kawashima et al. (25) & 2011 & 1998-2008 & 25 & 53 & 36 \\
\hline Bölükbas et al. (24) & 2012 & 1999-2008 & 107 & - & 47 \\
\hline Own data & 2017 & 2001-2016 & 46 & 44 & 33 \\
\hline
\end{tabular}

RCC, renal cell carcinoma; OS, overall survival.

ages were 6-11 years lower in the surgical series, ranging from 57.7-62.0 years, potentially explaining the marginally lower OS rates observed in our study (17-19,21-23,25-27). Hence, SBRT for pulmonary metastases might be a valid alternative at least for patients in higher age and with comorbidities.

A survival benefit for patients with oligo-metastatic RCC has been suggested when complete metastasectomy in parenchymal organs is performed compared to no surgical resection $(10,13)$. However, patient selection for this treatment approach is critical. A recent review of local treatments for metastases of RCC recommended the following criteria for suitable patients for a curative approach: good performance status, solitary or oligometastatic lesions, single organ sites, metachronous metastases, disease-free interval of over 2 years, absence of progression to treatment, and pulmonary metastasis size less than $4 \mathrm{~cm}$ in diameter in addition to several other, organ-specific factors (13). Corresponding to these recommendations, Karnofsky performance score and pulmonary metastasis diameter were identified as independent prognostic factors for superior OS in our study $(\mathrm{P}=0.010 ; \mathrm{P}=0.009)$. Furthermore, a trend towards improved OS was detected for metachronous lesions when compared to synchronous metastases $(\mathrm{P}=0.055)$. Patients analyzed in the current study were mainly treated with pulmonary SBRT in a potentially curable oligo-metastatic tumor state. Thirty-six patients $(83 \%)$ received definitive treatment including SBRT, surgery or radiotherapy with curative doses often in combination with systemic therapy to all detectable tumor lesions. However, 8 patients (17\%) were treated with SBRT for debulking and palliation of disease in combination with systemic therapy. This group of patients experienced a worse median survival of 19.6 months following SBRT compared to those patients treated with curative intent with 29.8 months $(\mathrm{P}=0.077$; Table 3). This finding may be explained by two mechanisms: (I) whether or not all lesions are radically treated maybe co-correlated with the overall metastatic tumor load, which is a known prognostic factor; (II) radical treatment of all imagingdefined metastases does indeed improve OS compared to debulking SBRT only.

RCC is considered to be an immunogenic tumor. Until 10 years ago, interferon alpha and high-dose interleukin-2 were the mainstays of treatment for patients with metastatic RCC (3). Although these systemic agents have been replaced by molecular, targeted therapies during the last years, the immunogenic potential of RCC remains an exciting avenue for future study. Immunotherapy with antibodies against the programmed cell death protein 1 (PD-1) or its ligand 1 (PD-L1) have demonstrated excellent preliminary results (48-50). Interestingly, SBRT might also be applied to enhance immunogenic the anti-tumor response via the abscopal effect: clinical studies have described regression of non-irradiated distant lesions following SBRT to an RCC metastasis (51-53). This effect is hypothesized to be immune 
mediated (40,53). However, the abscopal effect occurs rarely after SBRT alone and might be fostered by simultaneous treatment with synergistic immunomodulatory agents $(40,53)$. Hence, a potential future application of SBRT in patients with metastatic RCC might include the addition of systemic immunomodulatory therapy by inducing persistent anti-tumor immunity and potentially improving long-term survival $(40,53)$.

Limitations of this study were mainly caused by the retrospective nature of this analysis. Despite the extensive study timespan and multi-institutional approach, patient numbers were quite low as only few RCC patients are treated with SBRT to pulmonary metastases. The median follow-up time of 28.3 months for all patients was too short to assess long-term LC, survival and potentially late toxicity. Furthermore, systemic treatments have drastically changed over the course of this study, and their improved efficacy may have influenced LC and survival following SBRT.

\section{Conclusions}

SBRT especially with $\mathrm{BED}_{\text {iso }} \geq 130$ Gy for pulmonary RCC metastases resulted in excellent LC with only minimal acute and late toxicity. Survival was favorable for metastatic RCC patients. Future studies are needed to evaluate the potential of SBRT in combination with target molecular agents and/or immunotherapy in the treatment of oligo-metastatic RCC patients.

\section{Acknowledgements}

This work was supported by the Medical Faculty of Heidelberg University providing a research grant for J Hoerner-Rieber. We acknowledge the financial support of the Deutsche Forschungsgemeinschaft and Ruprecht-KarlsUniversität Heidelberg within the funding programme Open Access Publishing. Furthermore, we thank Michael Repka, Department of Radiation Medicine, Georgetown University Hospital, Washington, DC, USA for proofreading of the manuscript.

\section{Footnote}

Conflicts of Interest: The authors have no conflicts of interest to declare.

Ethical Statement: The analysis was approved by the Ethics committee of the University Hospital Heidelberg (S280/2014).

\section{References}

1. Ljungberg B, Bensalah K, Canfield S, et al. EAU guidelines on renal cell carcinoma: 2014 Update. Eur Urol 2015;67:913-24.

2. Ferlay J, Steliarova-Foucher E, Lortet-Tieulent J, et al. Cancer incidence and mortality patterns in Europe: Estimates for 40 countries in 2012. Eur J Cancer 2013;49:1374-403.

3. Choueiri TK, Motzer RJ. Systemic therapy for metastatic renal-cell carcinoma. N Engl J Med 2017;376:354-66.

4. Surveillance, Epidemiology, and End Results Programm. SEER stat fact sheets: kidney and renal pelvic cancer. Bethesda, MD: National Cancer Institute, 2017.

5. Altoos B, Amini A, Yacoub M, et al. Local control rates of metastatic renal cell carcinoma (rcc) to thoracic, abdominal, and soft tissue lesions using stereotactic body radiotherapy (SBRT). Radiat Oncol 2015;10:218.

6. Wersäll PJ, Blomgren H, Lax I, et al. Extracranial stereotactic radiotherapy for primary and metastatic renal cell carcinoma. Radiotherapy and Oncology 2005;77:88-95.

7. Jakubowski CD, Vertosick EA, Untch BR, et al. Complete metastasectomy for renal cell carcinoma: comparison of five solid organ sites. J Surg Oncol 2016;114:375-9.

8. Klatte T, Lam JS, Shuch B, et al. Surveillance for renal cell carcinoma: why and how? When and how often? Urol Oncol 2008;26:550-4.

9. Kyoda Y, Kobayashi K, Hirobe M, et al. Evaluation of long-term outcome for patients with renal cell carcinoma after surgery: analysis of cancer deaths occurring more than 10 years after initial treatment. Int J Clin Oncol 2014;19:146-51.

10. Macherey S, Kauffmann C, Heidenreich A, et al. Pulmonary metastasectomy in renal cell carcinoma. Urologe A 2017;56:1025-30.

11. Grimes NG, Devlin JM, Dunne DFJ, et al. A systematic review of the role of hepatectomy in the management of metastatic renal cell carcinoma. Eur J Surg Oncol 2014;40:1622-8.

12. Saitoh H. Distant metastasis of renal adenocarcinoma. Cancer 1981;48:1487-91.

13. Dabestani S, Marconi L, Hofmann F, et al. Local treatments for metastases of renal cell carcinoma: a systematic review. Lancet Oncol 2014;15:e549-61.

14. Heng DY, Rini BI, Garcia J, et al. Prolonged complete responses and near-complete responses to sunitinib in metastatic renal cell carcinoma. Clin Genitourin Cancer 2007;5:446-51. 
15. Motzer RJ, Hutson TE, Tomczak P, et al. Overall survival and updated results for sunitinib compared with interferon alfa in patients with metastatic renal cell carcinoma. J Clin Oncol 2009;27:3584-90.

16. Motzer RJ, Hutson TE, Tomczak P, et al. Sunitinib versus interferon alfa in metastatic renal-cell carcinoma. $\mathrm{N}$ Engl J Med 2007;356:115-24.

17. Hofmann HS, Neef H, Krohe K, et al. Prognostic factors and survival after pulmonary resection of metastatic renal cell carcinoma. Eur Urol 2005;48:77-81; discussion 81-2.

18. Murthy SC, Kim K, Rice TW, et al. Can we predict long-term survival after pulmonary metastasectomy for renal cell carcinoma? The Annals of Thoracic Surgery 2005;79:996-1003.

19. Pfannschmidt J, Hoffmann H, Muley T, et al. Prognostic factors for survival after pulmonary resection of metastatic renal cell carcinoma. Ann Thorac Surg 2002;74:1653-7.

20. Piltz S, Meimarakis G, Wichmann MW, et al. Long-term results after pulmonary resection of renal cell carcinoma metastases. Ann Thorac Surg 2002;73:1082-7.

21. Assouad J, Petkova B, Berna P, et al. Renal cell carcinoma lung metastases surgery: pathologic findings and prognostic factors. Ann Thorac Surg 2007;84:1114-20.

22. Kanzaki R, Higashiyama M, Fujiwara A, et al. Long-term results of surgical resection for pulmonary metastasis from renal cell carcinoma: a 25-year single-institution experience. Eur J Cardiothorac Surg 2011;39:167-72.

23. Meimarakis G, Angele M, Staehler M, et al. Evaluation of a new prognostic score (Munich score) to predict longterm survival after resection of pulmonary renal cell carcinoma metastases. Am J Surg 2011;202:158-67.

24. Bölükbas S, Kudelin N, Eberlein M, et al. The influence of the primary tumor on the long-term results of pulmonary metastasectomy for metastatic renal cell carcinoma. Thorac Cardiovasc Surg 2012;60:390-7.

25. Kawashima A, Nakayama M, Oka D, et al. Pulmonary metastasectomy in patients with renal cell carcinoma: a single-institution experience. Int J Clin Oncol 2011;16:660-5.

26. Kudelin N, Bolukbas S, Eberlein M, et al. Metastasectomy with standardized lymph node dissection for metastatic renal cell carcinoma: an 11-year single-center experience. Ann Thorac Surg 2013;96:265-70: discussion 270-1.

27. Renaud S, Falcoz PE, Alifano M, et al. Systematic lymph node dissection in lung metastasectomy of renal cell carcinoma: an 18 years of experience. J Surg Oncol 2014;109:823-9.

28. Wang CJ, Christie A, Lin M-H, et al. Safety and efficacy of stereotactic ablative radiation therapy for renal cell carcinoma extracranial metastases. Int J Radiat Oncol Biol Phys 2017;98:91-100.

29. Svedman C, Sandstrom P, Pisa P, et al. A prospective Phase II trial of using extracranial stereotactic radiotherapy in primary and metastatic renal cell carcinoma. Acta Oncol 2006;45:870-5.

30. Zelefsky MJ, Greco C, Motzer R, et al. Tumor control outcomes after hypofractionated and singledose stereotactic image-guided intensity-modulated radiotherapy for extracranial metastases from renal cell carcinoma. Int J Radiat Oncol Biol Phys 2012;82:1744-8.

31. Ranck MC, Golden DW, Corbin KS, et al. Stereotactic body radiotherapy for the treatment of oligometastatic renal cell carcinoma. Am J Clin Oncol 2013;36:589-95.

32. Stinauer MA, Kavanagh BD, Schefter TE, et al. Stereotactic body radiation therapy for melanoma and renal cell carcinoma: impact of single fraction equivalent dose on local control. Radiat Oncol 2011;6:34.

33. Teh B, Bloch C, Galli-Guevara M, et al. The treatment of primary and metastatic renal cell carcinoma (RCC) with image-guided stereotactic body radiation therapy (SBRT). Biomed Imaging Interv J 2007;3:e6.

34. Rieber J, Abbassi-Senger N, Adebahr S, et al. Influence of institutional experience and technological advances on outcome of stereotactic body radiation therapy for oligometastatic lung disease. Int J Radiat Oncol Biol Phys 2017;98:511-20.

35. Rieber J, Streblow J, Uhlmann L, et al. Stereotactic body radiotherapy (SBRT) for medically inoperable lung metastases-A pooled analysis of the German working group "stereotactic radiotherapy". Lung Cancer 2016;97:51-8.

36. Guckenberger M, Klement RJ, Allgauer M, et al. Local tumor control probability modeling of primary and secondary lung tumors in stereotactic body radiotherapy. Radiother Oncol 2016;118:485-91.

37. Timmerman R, McGarry R, Yiannoutsos C, et al. Excessive toxicity when treating central tumors in a phase II study of stereotactic body radiation therapy for medically inoperable early-stage lung cancer. J Clin Oncol 2006;24:4833-9.

38. Timmerman R, Paulus R, Galvin J, et al. Stereotactic body radiation therapy for inoperable early stage lung cancer. JAMA 2010;303:1070-6.

39. Park C, Papiez L, Zhang S, et al. Universal survival curve and single fraction equivalent dose: useful tools in understanding potency of ablative radiotherapy. Int $\mathrm{J}$ Radiat Oncol Biol Phys 2008;70:847-52. 
40. De Meerleer G, Khoo V, Escudier B, et al. Radiotherapy for renal-cell carcinoma. Lancet Oncol 2014;15:e170-7.

41. Tunio MA, Hashmi A, Rafi M. Need for a new trial to evaluate postoperative radiotherapy in renal cell carcinoma: a meta-analysis of randomized controlled trials. Ann Oncol 2010;21:1839-45.

42. Blanco AI, Teh BS, Amato RJ. Role of radiation therapy in the management of renal cell cancer. Cancers (Basel) 2011;3:4010-23.

43. Hallemeier CL, Choo R, Davis BJ, et al. Longterm outcomes after maximal surgical resection and intraoperative electron radiotherapy for locoregionally recurrent or locoregionally advanced primary renal cell carcinoma. Int J Radiat Oncol Biol Phys 2012;82:1938-43.

44. Walsh L, Stanfield JL, Cho LC, et al. Efficacy of ablative high-dose-per-fraction radiation for implanted human renal cell cancer in a nude mouse model. Eur Urol 2006;50:795-800; discussion 800.

45. De Bari B, Alongi F, Mortellaro G, et al. Spinal metastases: Is stereotactic body radiation therapy supported by evidences? Crit Rev Oncol Hematol 2016;98:147-58.

46. Kestin L, Grills I, Guckenberger M, et al. Dose-response relationship with clinical outcome for lung stereotactic body radiotherapy (SBRT) delivered via online image guidance. Radiother Oncol 2014;110:499-504.

47. Sterzing F, Brunner TB, Ernst I, et al. Stereotactic body radiotherapy for liver tumors: principles and practical

Cite this article as: Hoerner-Rieber J, Duma M, Blanck O, Hildebrandt G, Wittig A, Lohaus F, Flentje M, Mantel F, Krempien R, Eble MJ, Kahl KH, Boda-Heggemann J, Rieken S, Guckenberger M. Stereotactic body radiotherapy (SBRT) for pulmonary metastases from renal cell carcinoma-a multicenter analysis of the German working group "Stereotactic Radiotherapy". J Thorac Dis 2017;9(11):4512-4522. doi: 10.21037/jtd.2017.10.108 guidelines of the DEGRO Working Group on Stereotactic Radiotherapy. Strahlenther Onkol 2014;190:872-81.

48. Choueiri TK, Fishman MN, Escudier B, et al. Immunomodulatory activity of nivolumab in metastatic renal cell carcinoma. Clin Cancer Res 2016;22:5461-71.

49. Motzer RJ, Rini BI, McDermott DF, et al. Nivolumab for metastatic renal cell carcinoma: results of a randomized phase II Trial. J Clin Oncol 2015;33:1430-7.

50. Escudier B, Motzer RJ, Sharma P, et al. Treatment beyond progression in patients with advanced renal cell carcinoma treated with nivolumab in checkmate 025. Eur Urol 2017;72:368-76.

51. Ishiyama H, Teh BS, Ren H, et al. Spontaneous regression of thoracic metastases while progression of brain metastases after stereotactic radiosurgery and stereotactic body radiotherapy for metastatic renal cell carcinoma: abscopal effect prevented by the blood-brain barrier? Clin Genitourin Cancer 2012;10:196-8.

52. Wersall PJ, Blomgren H, Pisa P, et al. Regression of non-irradiated metastases after extracranial stereotactic radiotherapy in metastatic renal cell carcinoma. Acta Oncol 2006;45:493-7.

53. De Wolf K, Vermaelen K, De Meerleer G, et al. The potential of radiotherapy to enhance the efficacy of renal cell carcinoma therapy. Oncoimmunology 2015;4:e1042198. 